Research Paper

\title{
Effects of dexamethasone and nimesulide on bisphosphonate-related osteonecrosis of the jaw: An experimental study
}

\author{
Camila Carvalho de Oliveira ${ }^{\mathrm{a}, *}$, Paulo Goberlânio de Barros Silva ${ }^{\mathrm{a}}$, \\ Antonio Ernando Carlos Ferreira Jr. ${ }^{a}$, Romélia Pinheiro Gonçalves ${ }^{\mathrm{b}}$, Fabrício Bitu de Sousa ${ }^{\mathrm{a}}$, \\ Mário Rogério Lima Mota ${ }^{a}$, Ana Paula Negreiros Nunes Alves ${ }^{a}$ \\ a Department of Dental Clinic, Stomatology and Oral Pathology Sector, Federal University of Ceará, Fortaleza, Brazil \\ b Department of Pharmaceuticals Sciences, Federal University of Ceará, Fortaleza, Brazil
}

\section{A R T I C L E I N F O}

\section{Keywords:}

Bisphosphonate-associated osteonecrosis of the jaw

Anti-inflammatory agents

Dexamethasone

Nimesulide

\begin{abstract}
A B S T R A C T
Objective: To evaluate the effects of dexamethasone (DEX) and nimesulide (NIM) on Bisphosphonate-related Osteonecrosis of the Jaw (BRONJ) in rats.

Design: BRONJ was induced by zoledronic acid (ZA) infusion $(0.2 \mathrm{mg} / \mathrm{kg})$ in Wistar rats $(\mathrm{n}=8)$, followed by extraction of the left lower first molar (BRONJ groups). Control groups $(n=40)$ received saline (IV). For eight weeks, DEX $(0.04,0.4,4 \mathrm{mg} / \mathrm{kg}$ ) or saline (SAL) were administered by gavage $24 \mathrm{~h}$ before each infusion of ZA or saline (IV), or NIM (10.3 mg/kg) was administered $24 \mathrm{~h}$ and $12 \mathrm{~h}$ before each infusion of ZA or saline (IV). The haematological analyses were conducted weekly. After euthanasia (day 70), the jaws were submitted to radiographic and microscopic analysis. Kidney, liver, spleen and stomach were analysed histopathologically. Results: The BRONJ groups showed a higher radiolucent area compared with the control groups $(\mathrm{p}<0.05)$. Histomorphometric analysis revealed healing and new bone formation in the control groups, while the BRONJ groups exhibited devitalized bone with bacterial colonies and inflammatory infiltrate. The BRONJ-DEX 0.4 and $4 \mathrm{mg} / \mathrm{kg}$ groups had a greater number of bacterial colonies $(\mathrm{p}<0.05)$ and an increased polymorphonuclear cell count compared to the saline-BRONJ group, while the BRONJ-NIM group had a lower polymorphonuclear count ( $\mathrm{p}<0.05$ ). The BRONJ groups had leucocytosis, which was reduced by DEX administration. Treatments with DEX with or without ZA caused white pulp atrophy.

Conclusion: Thus, DEX or NIM therapy was not effective in preventing radiographic and histopathologic events associated with BRONJ. Treatment with DEX attenuated leucocytosis post-infusion with ZA.
\end{abstract}

\section{Introduction}

Medication-related Osteonecrosis of the Jaw (MRONJ) is defined as a necrotic bone exposure in the maxillofacial region that remains for more than eight weeks in patients treated with antiresorptive or antiangiogenic drugs (Ruggiero et al., 2014). One class of these drugs is the Bisphosphonates (BPs), which inhibit bone resorption mediated by osteoclasts and thus decrease bone turnover, increasing bone mineral density and reducing the risk of fractures. However, the suppressive effect of BPs reduces the renewal of bone tissue in the long term, which may contribute to the change in wound healing and induce necrosis. The latter is a common event in gnathic bone and is known as Bisphosphonate-related Osteonecrosis of the Jaw (BRONJ) (Baldi, Izziotti, Bonica, Pera, \& Pulliero, 2009; Walter et al., 2010)
The inflammatory process resulting from the extraction of teeth with previous periapical infections (Hoff et al., 2008; Kang et al., 2013; Marx, Sawatari, Fortin, \& Broumand, 2005; Ruggiero et al., 2009) and/ or teeth with periodontitis (Aghaloo et al., 2011; Ruggiero et al., 2009) has been shown to predispose BRONJ development. Furthermore, BRONJ development seems to be related to increased levels of pro-inflammatory mediators, such as interleukins, TNF- $\alpha$, IL-6, iNOS and IL$1 \beta$, which are pro-apoptotic for osteocytes (Barros-Silva et al., 2016; Deng, Tamai, Endo, \& Kiyoura, 2009; Mozzati et al., 2013; Tsao et al., 2013)

Dexamethasone (DEX) is a potent long-acting anti-inflammatory steroid with the ability to activate or inhibit transcription of some molecules, genes and inflammatory cytokines. (Beato, 1989; Falkenstein, Norman, \& Wehling, 2000; Mizuno et al., 1997) DEX, when

\footnotetext{
* Corresponding author at: Division of Oral Pathology, Department of Clinical Dentistry, School of Dentistry, Federal University of Ceará, Alexandre Barauna Street, 949, Rodolfo Teofilo, 60430-160, Fortaleza, Ceará, Brazil.

E-mail address: camilacdeoliv@gmail.com (C.C.d. Oliveira).
} 
used systemically and chronically, seems to increase the susceptibility to BRONJ development in various animal models (Kerachian, Harvey, Chow, Nahal, \& Séguin, 2011; López-Jornet, Camacho-Alonso, MolinaMiñano, Gómez-García, \& Vicente-Ortega, 2010; Sonis, Watkins, Lyng, Lerman, \& Anderson, 2009) and humans (Barasch et al., 2011; Chiu, Chiang, Chuang, \& Chang, 2010). However, the effect of subacute and different doses of this drug on BRONJ is not reported in the literature.

Nimesulide (NIM) is a non-steroidal anti-inflammatory (NSAID) with preferential selectivity for the inhibition of COX-2 2(Cullen, Kelly, Connor, \& Fitzgerald, 1998; Shah et al., 2001), and it is used in dentistry because it has a fast onset of action and is a good analgesic in dental (Ragot et al., 1994) and bone diseases, such as osteoarthritis (Bianchi \& Broggini, 2003). Furthermore, this drug has high activity against inflammation and fever, with a minor incidence of gastrointestinal effects compared to COX-1 selective NSAIDs (Bjarnason \& Thjodleifsson, 1999).

Considering inflammation as a potential process for triggering BRONJ, and knowing that it is an undefined pathogenesis condition without an established treatment protocol (Del Conte et al., 2010; Pozzi et al., 2007), it is important to investigate effective pharmacological mechanisms to prevent its development. In this context, it is hypothesized that administration of steroidal and non-steroidal anti-inflammatories could interfere with BRONJ development and may represent an effective preventive therapy. For this, the aim of this study is to evaluate the effects of dexamethasone and nimesulide on the tooth extraction site with chemonecrosis of the jawbone induced by zoledronic acid in rats.

\section{Materials and methods}

\subsection{Animals}

Eighty male Wistar rats (Rattus novergicus) with body weights between 180 and $280 \mathrm{~g}$ were selected. Animals were provided access to water and food ad libitum, housed under a light-dark cycle of $12 \mathrm{~h}$ at $22-24{ }^{\circ} \mathrm{C}$ and maintained in the same environmental conditions throughout the experiment.

The research project was approved by the Ethics Committee on Animal Use of the Federal University of Ceará (protocol 81/13) and it is in accordance with ARRIVE guidelines and The National Institutes of Health guide for the care and use of Laboratory animals (NIH Publications No. 8023, revised 1978).

\subsection{Induction of bisphosphonate-related osteonecrosis of jaw}

The osteonecrosis induction protocol was developed previously by this research group (Sonis et al., 2009). For this protocol, animals received weekly intravenous (IV) administration of three consecutive doses of zoledronic acid (ZA) $0.2 \mathrm{mg} / \mathrm{kg}$, followed by extraction of the left lower first molar 28 (twenty-eight) days after the last infusion of ZA (day 42). One week after surgery (day 49), another ZA dose $(0.2 \mathrm{mg}$ / $\mathrm{kg}$ ) was administered.

The animals were euthanized three weeks after the last ZA administration (day 70), followed by surgical excision of the jaw, stomach, kidneys, liver and spleen, which were fixed in buffered formalin 10\% (Fig. 1).

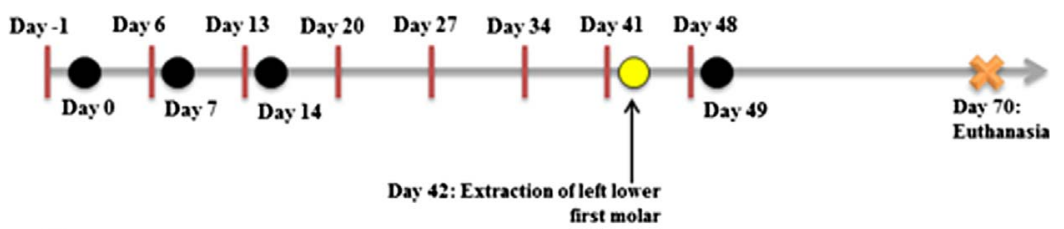

\subsection{Protocol of anti-inflammatory treatment (dexamethasone and nimesulide)}

The medium dose of dexamethasone was obtained from the antiinflammatory preemptive therapeutic dose indicated for dental surgical procedures in adult humans ( $4 \mathrm{mg}$, single dose, orally). This was converted to a dose in animals using the formula for transposition of dose based on body surface area recommended by Reigne and Blesch (Reigner \& Blesch, 2002). We obtained a dose of $0.4 \mathrm{mg} / \mathrm{kg}$, but in order to analyse the anti-inflammatory effect of an overdosage or a subtherapeutic dose, a curve was plotted with a dose ten times higher $(4 \mathrm{mg} / \mathrm{kg}$ ), and another with a dose ten times lower $(0.04 \mathrm{mg} / \mathrm{kg})$.

The establishment of the nimesulide therapeutic dose was determined as described above, although the dental surgery preemptive dose for adult humans is different (100 mg every $12 \mathrm{~h}$, orally) (Araújo et al., 2012; Avelar et al., 2012; Menezes and Cury, 2010). After conversion by the same formula, we obtained a value of $10.3 \mathrm{mg} / \mathrm{kg}$ of nimesulide (Fig. 1).

Dexamethasone, nimesulide or saline $(0.9 \%, 1 \mathrm{ml} / \mathrm{kg})$ were administered $24 \mathrm{~h}$ before the three intravenous administrations of ZA (days $-1,6,13$ ) by gavage, keeping this frequency weekly for 49 days until $24 \mathrm{~h}$ before the last dose of ZA (day 48). Only the animals treated with nimesulide received an additional dose $12 \mathrm{~h}$ after the first ZA intravenous administration (Fig. 1).

\subsection{Experimental groups}

The control animals consisted of five groups $(n=8)$ in which the left lower first molar was extracted and saline $0.9 \%$ was administered ( $1 \mathrm{ml} / \mathrm{kg}$, intravenously) in order to simulate ZA intravenous administration. In these groups, treatment by gavage was conducted with saline (SAL); dexamethasone 0.04, 0.4 or $4 \mathrm{mg} / \mathrm{kg}$ (DEX 0.04, 0.4 DEX, DEX 4, respectively); or nimesulide (NIM) $10.3 \mathrm{mg} / \mathrm{kg}$.

The BRONJ animals were divided into five groups $(n=8)$ and submitted to induction of osteonecrosis in the jaw with an intravenous injection of zoledronic acid. Then, we extracted the left lower first molar according to the described protocol. These groups were also treated by gavage with saline $(0.9 \%, 1 \mathrm{ml} / \mathrm{kg}$ ) (BRONJ-SAL), dexamethasone $(0.04 \mathrm{mg} / \mathrm{kg}, 0.4 \mathrm{mg} / \mathrm{kg}$ and $4 \mathrm{mg} / \mathrm{kg}$ ) (BRONJ-DEX 0.04; BRONJ-DEX 0.4; BRONJ-DEX-4, respectively) or nimesulide (10.3 mg/ $\mathrm{kg})$.

\subsection{Analysis of surgical difficulty}

The time required to perform the tooth extraction was timed, and the teeth were evaluated by an assistant for the number of fractured roots as a proxy for the surgical difficulty (Conte-Neto et al., 2013).

\subsection{Digital radiographic analysis (mandibles)}

After euthanasia, surgically excised jaws were radiographed using a conventional X-ray machine (DabiAtlante $63 \mathrm{Kvp}, 8 \mathrm{~mA}$ ) coupled with a Digora1 digital image capturing system. The radiographic technique employed was parallelism (long cone), with a distance of $10 \mathrm{~cm}$ and an exposure time of $0.18 \mathrm{~s}$ for all samples. Radiographs were 
quantitatively analysed using Image $J^{\circ}$. After randomization, an operator blindly and manually measured the radiolucent area in triplicate using a freehand selection approach. Through measure command, the total osteolytic demarcated area was obtained. The average of three measurements was designated the sample unit (Bodner, Kaffe, Littner, \& Cohen, 1993; Silva et al., 2015).

\subsection{Slide preparation and histomorphometric analysis}

The mandibles were decalcified in suspension (10\% EDTA, pH 7.3, $\mathrm{NaOH}, \mathrm{PA}$ ) (Keklikoglu, 2004; Silva et al., 2015), for four weeks. Subsequently, the material was submitted to histological processing to make microscopic slides stained by haematoxylin-eosin (HE).

After descriptive analysis, the histological slides were photographed in 10 (ten) microscopic fields at a high magnification $(400 \times)$ in the site corresponding to the dental socket of the first lower left molar and analysed by an operator who did not know which animal belonged to which group. Fields were selected starting always at the anterior border of the site post-extraction and following to the posterior side in order to not repeat the areas photographed.

The images were released in Image $J^{\circ}$ software, and through the cell counter command, the number of gaps of empty osteocytes and viable cells (Junqueira \& Junqueira, 1983), the number of osteoclasts with histological signs of vitality and apoptosis (nuclear hypo- or hyperchromatism and presence of multiple intracytoplasmic vacuoles) (Halleen et al., 1999; Hansen, Kirkpatrick, Walter, \& Kunkel, 2006; Pazianas, 2011) and the number of polymorphonuclear neutrophils (Silva et al., 2015) were evaluated. The sum of the fields of each slide was considered as the sampling unit, and the percentages of empty gaps of osteocytes and apoptotic osteoclasts were used for quantitative evaluation. In addition, the percentage of animals with bacterial colonies in the jawbone was recorded.

\subsection{Blood collection and analysis of haematological parameters}

Blood samples $(2 \mathrm{ml})$ were collected from the retro-orbital plexus of the animal with the aid of glass capillaries on day -1 prior to the administration of the anti-inflammatory and maintained weekly until day 69.

These samples were stored in $0.2 \mathrm{ml}$ of ethylenediaminetetraacetic acid (EDTA) disodium solution (1\%) and submitted to haematological analysis using an automated method for impedance principle (Sysmex KX 21N, Roche ). Erythrocytes (total number of red cells, haemoglobin, haematocrit and anisocytosis index (RDW)), number of platelets and the total number of leukocytes and lymphocytes were measured quantitatively.

\subsection{Analysis of toxicity parameters in the different experimental groups}

Liver, kidneys, spleen and stomach were removed on the day of euthanasia and fixed in buffered formalin (10\%). After a minimum of $24 \mathrm{~h}$, macroscopic analysis was performed and sample fragments were used to make microscope slides by staining with haematoxylin-eosin (Junqueira \& Junqueira, 1983). Slide analysis was conducted in an optical microscope to verify the presence of specific toxicity changes in each body.

\subsection{Statistical analysis}

Quantitative data were submitted to the Kolmogorov-Smirnov normality test. For parametric data, ANOVA, one-factor or multifactor paired or unpaired associated with Bonferroni post-testing was used, depending on the indication, and the results were expressed as the mean \pm SEM.

The area under the curve was calculated for the analysis of haematological parameters (red blood cells, haemoglobin, haematocrit, anisocytosis index, platelets, leukocytes, lymphocytes) of each animal, for which the mean and standard deviation for each group were calculated. From these data, one-factor ANOVA followed by the Bonferroni post-test was calculated.

Additionally, the presence/absence of toxicity was expressed by absolute frequency (percentage) and analysed by the chi-square test.

All analyses were performed using GraphPad Prism1 5.0 statistical software, using a significance level of 95\% ( $\mathrm{p}<0.05)$.

\section{Results}

\subsection{Analysis of surgical difficulty}

The time to perform tooth extraction was statistically similar between the groups that received ZA intravenous administration (IV) and the controls, which received saline IV ( $\mathrm{p}=0.791,2$-way ANOVA/ Bonferroni). However, the group submitted to BRONJ and treated with the highest dose of DEX $(4 \mathrm{mg} / \mathrm{kg})(136.8 \pm 12.0 \mathrm{~s})$ needed more time for the extraction of the first molar compared to BRONJ-SAL $(88.6 \pm 6.3 \mathrm{~s})(\mathrm{p}<0.01,1$-way ANOVA/Bonferroni) (Fig. 2A).

There was no significant difference in fracture number occurring during the tooth extraction in the BRONJ groups (BRONJ-SAL, BRONJDEX 0.04, BRONJ-DEX 0.4, BRONJ-DEX 4, BRONJ-NIM) compared to the control (SAL DEX 0.04, 0.4 DEX, DEX 4 and NIM) ( $p=0.139,2$ way ANOVA/Bonferroni), showing the similarity of surgical damage in all animals (Fig. 2B).

\subsection{Digital radiographic analysis (mandibles)}

The suggestive osteolytic area of necrosis and/or non-healing bone measured from digital radiographs of mandibles was significantly higher in the groups receiving ZA (IV) compared to the control groups ( $\mathrm{p}<0.01 ; 2$-way ANOVA/Bonferroni; Mean \pm SEM) (Fig. 3). No differences were seen between the radiolucent areas in BRONJ groups treated with DEX (BRONJ-DEX 0.04; BRONJ-DEX 0.4; BRONJ-DEX 4) or NIM (BRONJ-NIM) compared to the BRONJ group that received saline by gavage (BRONJ-SAL) $(\mathrm{p}=0.896,1$-way ANOVA/Bonferroni) (Fig. 3).

\subsection{Histopathological and histomorphometric analysis of mandibles}

Histopathological analysis of the tooth extraction sites of the control groups (SAL, DEX 0.04, 0.4 DEX, DEX 4, NIM), which received only saline (IV), revealed organized and cellularized bone with osteoblast activity and rare osteoclasts without histological changes as well as no residual inflammatory foci.

We also observed dense fibrous connective tissue covered by stratified squamous epithelium. In the groups submitted to BRONJ (BRONJ-SAL, BRONJ-DEX 0.04, BRONJ-DEX 0.4, BRONJ-DEX 4, BRONJ-NIM), bone trabeculae showed many empty osteocyte gaps, with apoptotic osteoclasts (pyknotic nucleus and vacuolation) and areas of intense inflammation, consisting predominantly of polymorphonuclear neutrophils (Fig. 4).

Histomorphometric analysis of the mandible bone trabeculae was used to evaluate the percentage of apoptotic osteoclasts and osteocytes with empty lacunae, the number of animals with bacterial colonies and the number of polymorphonuclear neutrophils.

The mandibles of the BRONJ groups [BRONJ-SAL (56.1 $\pm 6.5 \%)$, BRONJ-DEX 0.04 (88.7 \pm 3.4\%), BRONJ-DEX 0.4 (90.5 $\pm 4.6 \%$ ), BRONJ-DEX 4 (95.2 $\pm 2.1 \%)$, BRONJ-NIM (92.8 $\pm 7.1 \%)]$ showed a higher percentage of apoptotic osteoclasts when compared with the respective control groups [SAL (0.7 $\pm 0.7 \%)$, DEX $0.04(3.7 \pm 1.8 \%)$, DEX $0.4 \quad(15.3 \pm 7.4 \%), \quad$ DEX $4 \quad(12.5 \pm 8.0 \%), \quad$ NIM $(16.7 \pm 16.7 \%)] \quad(\mathrm{p}<0.0001, \quad 2$-way ANOVA/Bonferroni, Mean \pm SEM) (Fig. 5A). Treatment with DEX or NIM associated with ZA (IV) (BRONJ-DEX 0.04, BRONJ DEX 0.4, BRONJ-DEX 4, BRONJ- 


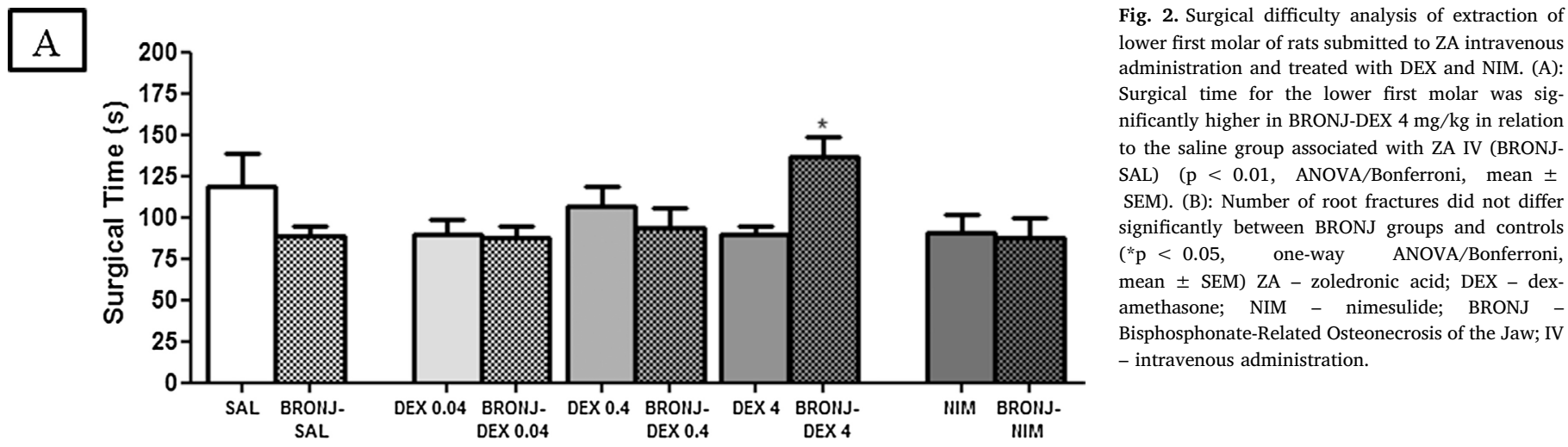

$\mathrm{B}$

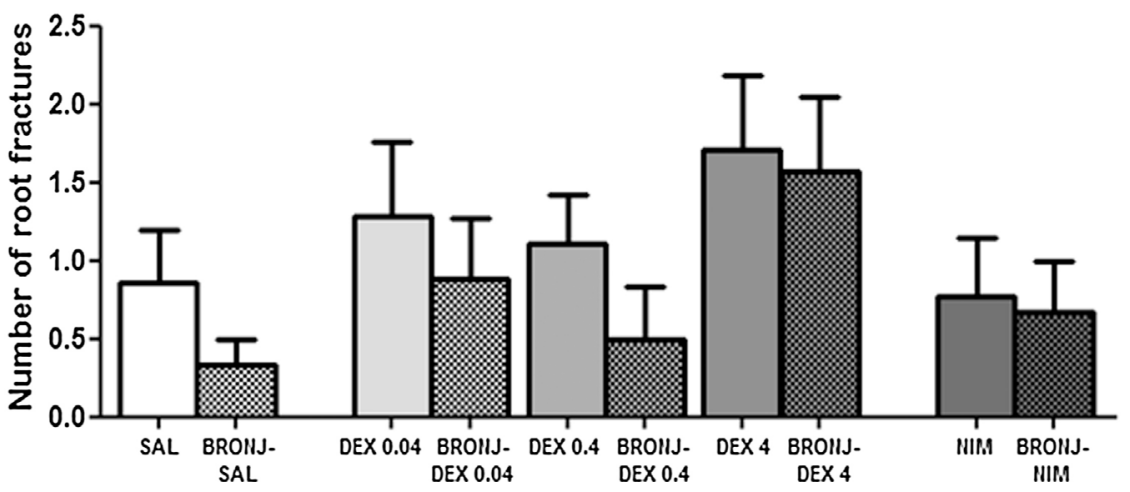

Fig. 2. Surgical difficulty analysis of extraction of lower first molar of rats submitted to ZA intravenous ministration and treated with DEX and NIM. (A) ar was sig$\mathrm{SAL}) \quad(\mathrm{p}<0.01$, ANOVA/Bonferroni, mean \pm (B): Number of root fractures did not differ ANOVA/Bonferroni, mean \pm SEM) ZA - zoledronic acid; DEX - dexamethasone; NIM - nimesulide; BRONJ - intravenous administration. Bisphosphonate-Related Osteonecrosis of the Jaw; IV
NIM) significantly increased the percentage of apoptotic osteoclasts compared to the BRONJ group treated only with saline (BRONJ-SAL) ( $p<0.0001,2$-way ANOVA/Bonferroni; Mean \pm SEM) (Fig. 5A).

Groups submitted to BRONJ [BRONJ-SAL (55.6 $\pm 8.6 \%)$, BRONJDEX 0.04 (54.0 $\pm 7.1 \%)$, BRONJ-DEX $0.4(60.8 \pm 12.5 \%)$, BRONJDEX $4(70.3 \pm 5.6 \%)$, BRONJ-NIM $(52.0 \pm 12.8 \%)]$ had a significantly higher percentage of empty osteocyte lacunae compared to the respective control groups [SAL $(1.8 \pm 0.4 \%)$; DEX 0.04 (4.2 $\pm 0.6 \%)$; DEX 0.4 (3.0 $\pm 0.7 \%)$, DEX 4 (2.3 $\pm 0.8 \%)$, NIM $(3.6 \pm 1.4 \%)] \quad(\mathrm{p}<0.0001, \quad 2$-way $\quad$ ANOVA/Bonferroni, Mean \pm SEM) (Fig. 5B).

The control groups (SAL; DEX 0.04; DEX 0.4; DEX 4, NIM) showed no bacterial colonies at the tooth extraction site, while in all BRONJ groups (BRONJ-SAL; BRONJ-DEX 0.04; BRONJ-DEX 0.4; BRONJ-DEX 4) basophilic amorphous material consistent with bacterial colonies was observed ( $\mathrm{p}<0.05$, chi-square) (Fig. 5C). Among the BRONJ groups, animals treated with the two higher doses of dexamethasone [BRONJ-DEX 0.4 (5; 100\%); BRONJ-DEX 4 (7; 100\%)] showed a greater and significant percentage of animals with bacterial colonies compared with the group submitted to BRONJ treated with saline
[BRONJ-SAL (4; 50\%)] (p < 0.05, Chi-square) (Fig. 5C).

The number of polymorphonuclear neutrophils revealed that groups submitted to BRONJ and treated with saline [BRONJ-SAL $(439.4 \pm 126.0)$ ] or dexamethasone [BRONJ-DEX 0.04 (595.8 \pm 145.5); BRONJ-DEX 0,4 (759.2 \pm 238.2); BRONJ-DEX 4 (747.7 \pm 171.5$)]$ had a higher number of polymorphonuclear neutrophils when compared to the respective control groups [SAL (0.8 \pm 0.6); DEX 0.04 (2.3 \pm 0.7), DEX 0.4 (20.0 \pm 6.3), DEX 4 $(59.4 \pm 21.3)] \quad(\mathrm{p}<0,0001, \quad 1$-way ANOVA/Bonferroni, Mean \pm SEM) (Fig. 5D). However, the group submitted to BRONJ and treated with nimesulide [BRONJ-NIM $(410.8 \pm 359.3)]$ showed no significant changes compared to the control group [NIM $(51.7 \pm 15.8)]$ but presented a significant reduction in the number of polymorphonuclear neutrophils compared to the BRONJ-SAL group ( $\mathrm{p}<0,05$, 1-way ANOVA/Bonferroni, Mean \pm SEM) (Fig. 5D). Groups submitted to BRONJ and treated with dexamethasone (BRONJDEX 0.04; BRONJ-DEX 0.4; BRONJ-DEX 4) revealed a greater number of polymorphonuclear neutrophils in relation to the BRONJ-SAL group ( $\mathrm{p}<0.05,1$-way ANOVA/Bonferroni, Mean \pm SEM).

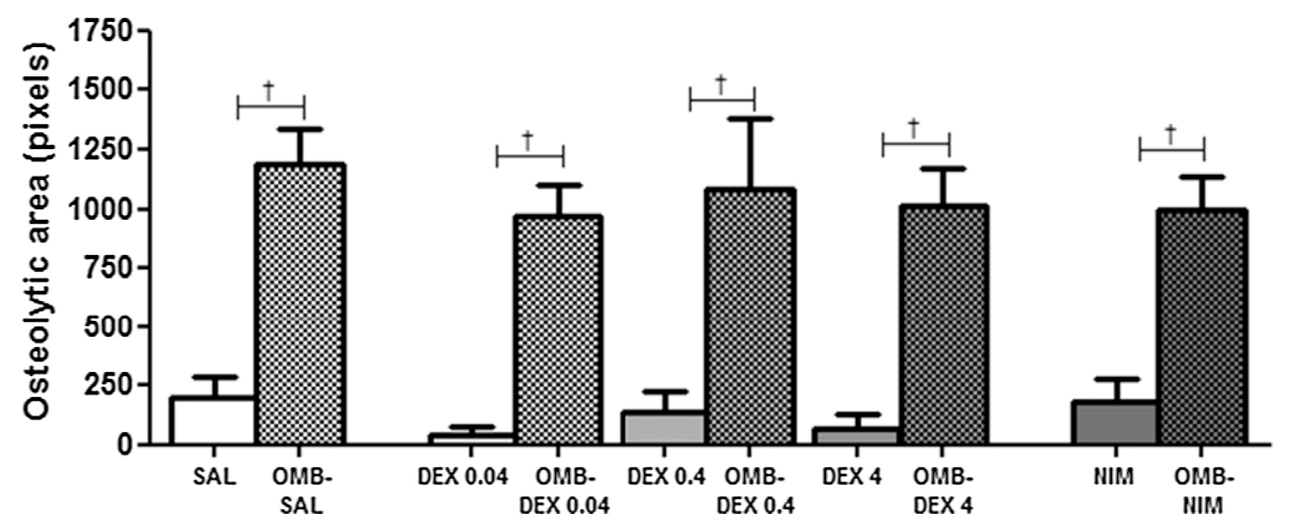

Fig. 3. Osteolytic area analysis, in pixels, on extraction site of the lower first molar at the 70th day in the control groups (without ZA) and in the BRONJ rats (submitted to ZA intravenous administration) treated with saline, DEX or NIM. The osteolytic area was significantly higher in the BRONJ groups in relation to the respective control groups. However, there were no statistically significant differences between the BRONJ rats treated with DEX $(0.04,0.4$ or $4 \mathrm{mg} / \mathrm{kg})$ or NIM $(10.3 \mathrm{mg} / \mathrm{kg})$ and the corresponding group of saline intravenous administration. ( $\mathrm{p}$ p $<0.01, \quad$ 2-way ANOVA/Bonferroni, mean \pm SEM). ZA - zoledronic acid; DEX - dexamethasone; NIM - nimesulide; BRONJ Bisphosphonate-Related Osteonecrosis of the Jaw. 


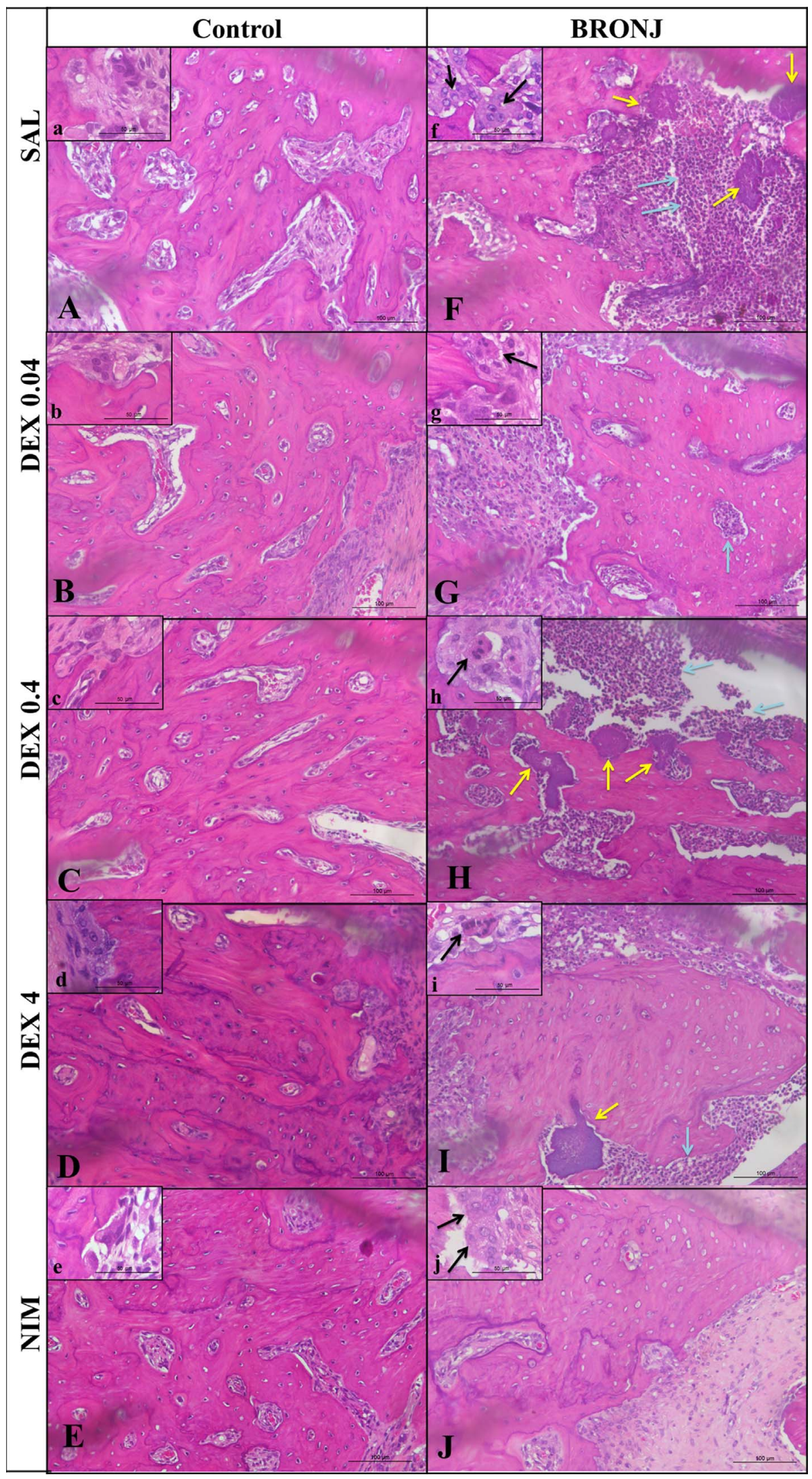

Fig. 4. Photomicrograph of extraction site of lower first molar in rats of the control groups (without ZA) and in the BRONJ rats (submitted to ZA intravenous administration) treated with saline, DEX or NIM. A-E, a-e: Control groups treated with saline, DEX or NIM (A-E): Tooth extraction site exhibiting bone trabeculae with high cellularity, filled by osteocytes and no inflammatory infiltrate. (a-e): On the detail, there are typical osteoclasts. F-f: BRONJ-Saline Group; G-g: BRONJ-DEX $0.04 \mathrm{mg} / \mathrm{kg}$ group; H-h: BRONJDEX $0.4 \mathrm{mg} / \mathrm{kg}$ group; I-i: BRONJ-DEX $4 \mathrm{mg} / \mathrm{kg}$ groups Tooth extraction sites show empty osteocyte lacunae, devitalized bone tissue, intense neutrophilic inflammatory infiltrate (blue arrows) and bacterial colonies (yellow arrows). In the detail, apoptotic osteoclasts near necrotic bone tissue are observed, sometimes with pyknosis (black arrows) (f-h). J-j: BRONJ-NIM $10.3 \mathrm{mg} / \mathrm{kg}$ group - Tooth extraction site demonstrates empty osteocyte lacunae, trabeculae necrosis and presence of numerous osteoclasts, revealing cytoplasmic vacuolization (black arrows), on the detail (j). Haematoxylin-eosin $(200-400 \times)$. ZA - zoledronic acid; DEX - dexamethasone; NIM - nimesulide; BRONJ Bisphosphonate-Related Osteonecrosis of the Jaw. (For interpretation of the references to colour in this figure legend, the reader is referred to the web version of this article.)

\subsection{Analysis of haematological parameters}

Groups that received ZA intravenous administration (BRONJ-SAL, BRONJ-DEX 0.04; BRONJ-DEX 0.4; BRONJ-DEX 4, BRONJ-NIM) had a lower area under the curve for the red blood cell count compared to the respective control groups (SAL; DEX 0.04; DEX 0.4; DEX 4; NIM) ( $\mathrm{p}<0.0001$, 1-way ANOVA/Bonferroni, Mean \pm SEM). Furthermore, the area under the curve calculated for the anisocytosis 


\section{A}

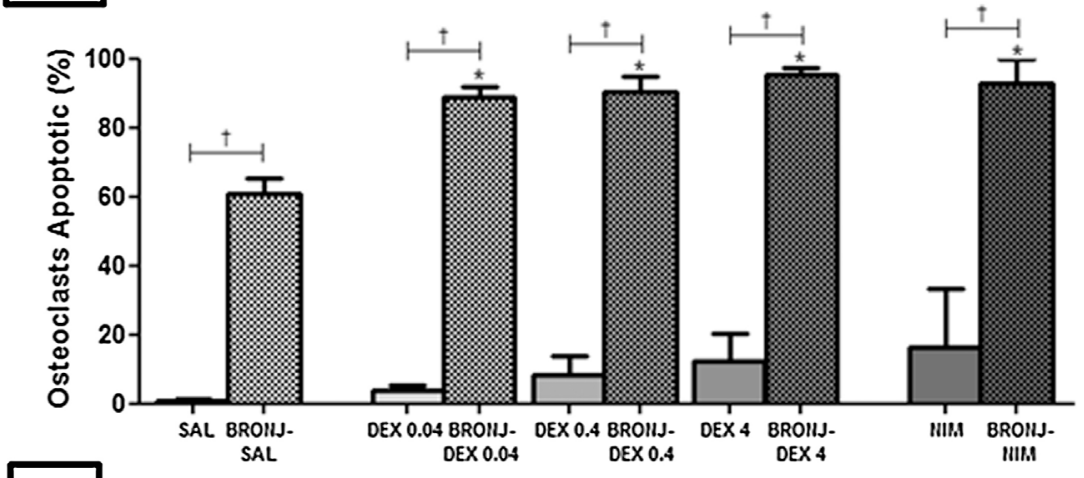

B

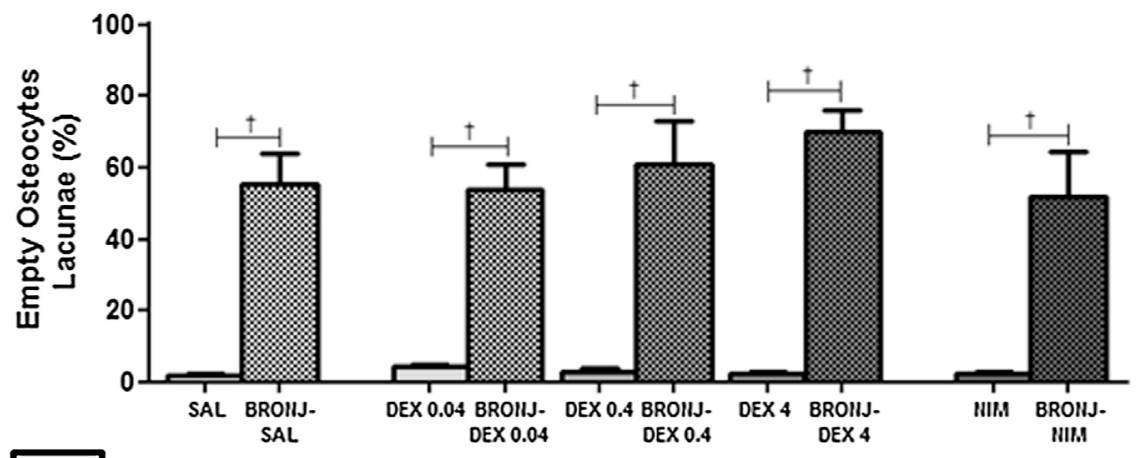

$\mathrm{C}$

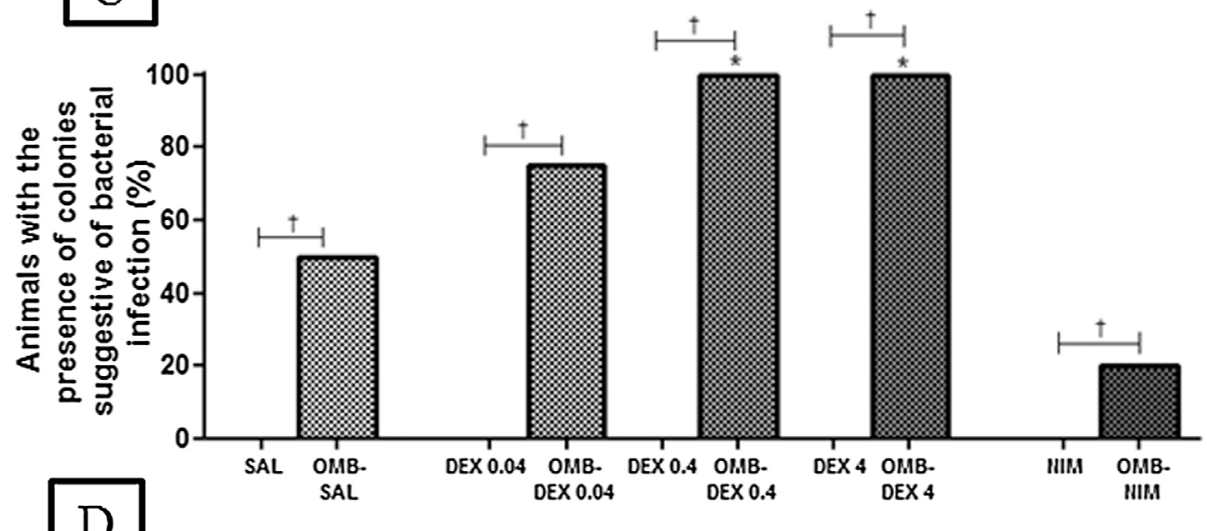

\section{$\mathrm{D}$}

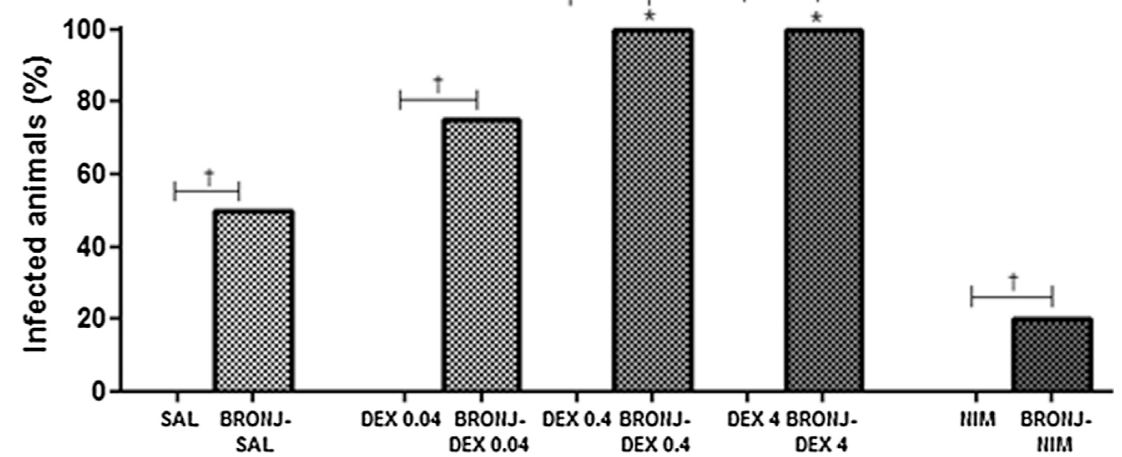

Fig. 5. Histomorphometric analysis of the extraction site of the lower first molar in rats of the control groups (without $\mathrm{ZA}$ ) and in the BRONJ rats (submitted to ZA intravenous administration) treated with saline, DEX or NIM. BRONJ groups presented a significant increase in apoptotic osteoclast percentage ( $\mathrm{p}<0.0001)$ (A), empty osteocyte lacunae (B), number of animals with bacterial colonies (C) and polymorphonuclear neutrophil count (D) in relation to the control groups. The BRONJ groups treated with an anti-inflammatory showed a higher percentage of apoptotic osteoclasts in relation to the BRONJ-saline group ( $\mathrm{p}<0.0001)$. The BRONJ-groups treated with DEX $(0.4$ and $4 \mathrm{mg} / \mathrm{kg}$ ) showed a higher percentage of animals with bacterial colonies and a significant increase in polymorphonuclear neutrophil count at the tooth extraction sites when compared to the saline control group (BRONJ$\mathrm{SAL}$ ). Rats treated with NIM $10,3 \mathrm{mg} / \mathrm{kg}$ showed a reduction in polymorphonuclear neutrophil count compared to the BRONJ-SAL group ( $\left.{ }^{*} \mathrm{p}<0.05\right)$ (mean \pm SEM, oneway ANOVA/Bonferroni) and when compared to the control group ( $\dagger \mathrm{p}<0.05$ ) (mean \pm SEM; 2-way ANOVA/ Bonferroni). ZA - zoledronic acid; DEX - dexamethasone; NIM - nimesulide; BRONJ - Bisphosphonate-Related Osteonecrosis of the Jaw. 
( $\mathrm{p}<0.0001,1$-way ANOVA/Bonferroni, Mean \pm SEM). It is possible that the increase in leukocytes observed in the groups receiving ZA intravenous administration was caused by the higher number of lymphocytes, because this parameter had a greater area under the curve compared to their respective control ( $\mathrm{p}<0.0001,1$-way ANOVA/ Bonferroni, Mean \pm SEM) (Table 1).

Animals of the control groups and of the BRONJ groups treated with dexamethasone 0.4 and $4 \mathrm{mg} / \mathrm{kg}$ (DEX 0.4; DEX 4; BRONJ-DEX 0.4; BRONJ-DEX 4) had a reduction in total leukocytes in relation to the respective saline groups (SAL; BRONJ- SAL) ( $p<0.0001$, 1-way ANOVA/Bonferroni, Mean \pm SEM). In these animals, we observed a reduction in the area under the curve calculated for lymphocyte number in a dose-dependent manner, suggesting that leukopenia caused by the administration of DEX occurred by lymphocyte reduction (p < 0.0001, 1-way ANOVA/Bonferroni, Mean \pm SEM) (Table 1).

\subsection{Analysis of toxicity parameters in different experimental groups}

The morphological evaluation of the stomach in all experimental groups showed preservation of the gastric mucosa. However, the kidneys of animals that received intravenous administration of zoledronic acid, regardless of the treatment (saline or dexamethasone or nimesulide), had a higher frequency of hyaline-cylinders in renal tubules (proximal and distal). Only in the livers from groups treated with nimesulide was a focal necrosis of hepatocytes observed although without interstitial fibrosis.

Animals treated with higher doses of dexamethasone (DEX 0.4; DEX 4; BRONJ-DEX 0.4; BRONJ-DEX 4)] had splenic atrophy of the white pulp compared to the saline control (SAL; BRONJ-SAL).

\section{Discussion for an original paper}

In this study, we investigated whether the suppression of inflammation via the use of steroidal and nonsteroidal anti-inflammatories would prevent or mitigate BRONJ. It was observed that no anti-inflammatory treatment acted effectively to prevent this event, with classic histological and radiographic signs of BRONJ in all groups submitted to ZA intravenous administration. The treatment with nimesulide, although it did not attenuate bone necrosis, was able to effectively reduce neutrophil migration to the site of tooth extraction. These findings indicate that inflammation suppression through the weekly administration of nimesulide and dexamethasone in different doses was not decisive for BRONJ development.

The evaluation of surgical difficulty by the number of root fractures during the tooth extraction showed no significant differences among any of the groups. Similar results were reported in the literature by other authors. Maahs et al. (Maahs, Azambuja, Campos, Salum, \& Cherubini, 2011) found no differences in the number of fractures among Wistar rats submitted or not to zoledronic acid treatment, with different schemes and routes of administration of aminobisphosphonates. In contrast, studies with other lineages of rats (Conte-Neto, De Souza Bastos, Spolidorio, Chierici Marcantonio, \& Marcantonio, 2012) and beagle dogs (Allen, Chu, \& Ruggiero, 2013) showed that there are more fractures after treatment with bisphosphonates. The authors explained this finding through increased bone density and reduction of bone elasticity derived by bone antiresorptive treatment coupled with excessive surgical force (Conte-Neto et al., 2012).

Reflecting the surgical difficulty parameter, the time taken to perform the extraction was significantly higher in the BRONJ 4-DEX group compared to the BRONJ-SAL group. However, there were no significant differences when comparing the control groups and the animals submitted to BRONJ. It was noted that the rib resistance in dogs treated with the combination of zoledronic acid and dexamethasone was higher than in animals that received only zoledronic acid, which suggests an influence of dexamethasone on bone turnover (Ma \& Wang, 2014). The ribs, in addition to the jawbone, have shown changes in mechanical 
properties associated with bisphosphonate treatment because the rib is a cortical bone which undergoes a considerable amount of bone remodelling (Allen, Reinwald, \& Burr, 2008; Ruggiero et al., 2014).

Radiographic evidence of BRONJ generally consists of radiolucent irregular areas with occasional sequestrum with little evidence of healing and may also present pathological fractures (Chiandussi et al., 2006; Krishnan, Arslanoglu, Yildirm, Silbergleit, \& Aygun, 2009). Sonis et al. (Sonis et al., 2009), in a model that mimics BRONJ in SpragueDawley rats, observed an absence of radiopacity in the jaws of animals submitted to a weekly regimen of zoledronic acid from digital radiographs, which did not differ between groups treated with the co-administration of dexamethasone, suggesting little or no influence of the administration of this drug in radiographic changes. These findings corroborate the results of this present study, where we observed higher osteolytic areas in digital radiography of animals receiving ZA (BRONJ groups); however, we did not see increased lytic areas in the animals treated with anti-inflammatories. The histomorphometric study examined different parameters, which together confirmed the presence of necrotic bone in post-extraction sites. All control animals treated with saline IV exhibited cellularized bone, with osteoblasts and osteoclasts without histologic changes, and an absence of signs suggestive of infection, with low numbers of polymorphonuclear neutrophils, similar to previous studies (Bi et al., 2010; Marino et al., 2012; Sonis et al., 2009).

The groups treated with ZA showed low bone cellularity, with empty osteocyte lacunae, apoptotic osteoclasts, bacterial colonies consistent with Actinomyces and polymorphonuclear neutrophils, confirming the presence of sequestrum and histologic changes of BRONJ. These microscopic findings were also described by other authors in experimental models of BRONJ (Bi et al., 2010; Conte-Neto et al., 2013; Marino et al., 2012; Sonis et al., 2009) and necrotic bone associated with bisphosphonate therapy in humans (Favia, Pilolli, \& Maiorano, 2009).

In general, BRONJ-DEX animals $(0.04 \mathrm{mg} / \mathrm{kg} ; 0.4 \mathrm{mg} / \mathrm{kg} ; 4 \mathrm{mg} / \mathrm{kg})$ and BRONJ-NIM animals revealed histopathological features similar to the BRONJ-SAL group. This demonstrated that the therapeutic schemes of nimesulide and dexamethasone (weekly) were unable to prevent the development of this condition. Bi et al. (Bi et al., 2010) also found no association between inflammation and BRONJ, except when an immunosuppressant was used in association with aminobisphosphonate, such as high-dose dexamethasone and docetaxel, where they showed the presence of inflammatory infiltrate. In agreement with this result, bacterial colonies and polymorphonuclear neutrophils were observed in greater numbers in the BRONJ-DEX $0.4 \mathrm{mg} / \mathrm{kg}$ and BRONJ-DEX $4 \mathrm{mg}$ / $\mathrm{kg}$ groups than in the BRONJ-SAL group.

The high presence of bacterial colonies in animals treated with dexamethasone may be due to the chronic scheme of drug administration over eight weeks, making the corticoid capable of eliciting immunosuppression in animals. In addition to this chronic scheme, in this study we used weekly dexamethasone administration (intramuscularly), which is similar to experimental models of osteonecrosis-related steroids reported by Ma et al. (Ma \& Wang, 2014) and Tian et al. (Tian, Wen, Dang, Fan, \& Wang, 2014), which demonstrates that this period is sufficient for the chronic effects of corticosteroids to appear. On the other hand, Kobayashi et al. (Kobayashi et al., 2010) showed that infection seems to be associated with ZA administration via an increase in bacterial activity and the growth of an oral biofilm. Interestingly, Bi et al. (Bi et al., 2010) also observed an increase in the inflammatory foci in the tooth extraction site in the jaws of rats treated with zoledronic acid and dexamethasone compared to in the animals treated with isolated aminobisphosphonate.

Other authors attribute immunosuppression as the cause of a failed host reaction against the presence of non-reabsorbed bone fragments colonized by bacteria, which increases the chance of infection and bone exposition. Bone fragments are easily exposed into the external surface of the mucosa and readily colonized by bacteria from the oral environment. This process can amplify infection and bone exposure, generating an increase in local inflammatory infiltrate (Jabbour, Hakim, Henderson, \& Albuquerque, 2014).

Nimesulide is a drug with anti-inflammatory and antioxidant actions, reducing neutrophil phagocytic capacity (Bravo-Cuellar, GarcíaReyes, Barba-Barajas, Carranco-López, \& Dominguez-Rodriguez, 2003) by inhibition of superoxide anion synthesis and consequently lowering the oxidative potential (Capsoni et al., 1987; Mello, Laurindo, \& Cossermelli, 1994; Ottonello, Dapino, Pastorino, \& Dallegri, 1992). Moreover, nimesulide can reduce in vitro the chemotaxis of polymorphonuclear neutrophils stimulated at the site of inflammation (Mello et al., 1994) and adhesion and transmigration through endothelial cells activated by cytokines (Dapino, Ottonello, \& Dallegri, 1994). These properties can explain the results of the present research, where a smaller number of polymorphonuclear neutrophils in the tooth extraction site were observed for the BRONJ-NIM animals than the BRONJ-SAL group.

Animals submitted to BRONJ and treated with anti-inflammatory drugs (BRONJ-DEX $0.04 \mathrm{mg} / \mathrm{kg}$; BRONJ-DEX $0.4 \mathrm{mg} / \mathrm{kg}$, BRONJ-DEX $4 \mathrm{mg} / \mathrm{kg}$; BRONJ-NIM) had a lower percentage of apoptotic osteoclasts compared to the BRONJ-SAL group. This effect may have occurred due to the reduction in inflammation promoted by the anti-inflammatory agent, which resulted in a decrease in osteoclastogenic mediators and a lower number of viable osteoclasts. The minor number of viable osteoclasts may reflect an imbalance between the number of normal and apoptotic osteoclasts (Tompkins, 2016).

In this study, none of the control animals treated with different doses of dexamethasone showed radiological or histological signs of osteonecrosis. These data are consistent with the findings of $\mathrm{Bi}$ et al. (Bi et al., 2010) Those authors administered dexamethasone treatment weekly ( $5 \mathrm{mg} / \mathrm{kg}$, intraperitoneally) in C57BL6 mice and verified that three weeks after tooth extraction most of the necrotic alveolar bone was replaced by viable spongy bone. After six weeks, they found complete replacement of necrotic lamellar bone tissue in the alveoli. In addition, therapy with dexamethasone $(3.8 \mathrm{mg} / \mathrm{kg} /$ weekly), an equivalent dose to that used in cancer patients ( $40 \mathrm{mg} /$ patient/week), was given to Sprague-Dawley rats after tooth extraction, and it did not interfere with dental alveoli healing (Jabbour et al., 2014). This dose was similar to the higher dose of dexamethasone used in this study.

Haematological analysis of the animals submitted to ZA intravenous administration showed a decrease in the number of red blood cells and increased anisocytosis index, and the treatment with different anti-inflammatory drugs did not appear to influence these parameters. Although it has been shown previously that the use of alendronate sodium in high doses has no cytotoxic effects on red blood cells (Kopka, Janiszewks, Szwed, Duda, \& Bukowska, 2011), a study that used a similar methodology to the present research showed that anaemic events may occur in animals receiving zoledronic acid at doses of 0.04, 0.20 and $1.00 \mathrm{mg} / \mathrm{kg}$ (Silva et al., 2015). A meta-analysis article from randomized clinical trials confirms that there is a significant and higher relative risk (1.33) of zoledronic acid treatment in the development of anaemia (Zhu et al.). However, more studies are needed to elucidate this relationship between ZA and anaemia.

In our research, mice that received zoledronic acid showed lymphocytosis when compared to those who were not given the bisphosphonate. It is known that aminobisphosphonates arose to modulate immunity in humans by inhibition of the mevalonate pathway that produces the accumulation of the intermediates isopentenyl diphosphate and dimethylallyl diphosphate in monocytes. Furthermore, it results in activation of T lymphocytes $\gamma \mathrm{d}$ (Roelofs, Thompson, Ebetino, Rogers, \& Coxon, 2010), which could explain the lymphocytosis found in the animals treated with ZA. On the other hand, dexamethasone has suppressive effects on white blood cells. In this study, the continued and single dexamethasone therapy in doses of 0.4 and $4 \mathrm{mg} / \mathrm{kg}$ for eight weeks in rats caused leukopenia. Moreover, in the animals that received zoledronic acid intravenous administration, dexamethasone therapy was effective in preventing lymphocytosis generated by 
bisphosphonates, which may show benefits for patients who already use this combination therapy.

The oral aminobisphosphonates, such as alendronate and risedronate, have been associated with adverse effects in the upper gastrointestinal tract (Khapra \& Rose, 2006). In the present study, we used zoledronic acid intravenous administration, which is not associated with changes in the gastric mucosa in the animals of the BRONJ groups.

The animals treated with nimesulide had livers with focal necrosis of hepatocytes but without interstitial fibrosis association. It is known that NSAIDs are classically related to liver injury, representing $43 \%$ of liver damage arising from drugs (Licata, Calvaruso, Cappelo, Craxi, \& Amasio, 2010). Nimesulide specifically shows potential hepatotoxicity through reactive metabolite formation that produces oxidative stress and mitochondrial damage (Mingatto et al., 2002). This mechanism could explain the liver damage in animals that received treatment with this drug. The zoledronic acid intravenous administration did not induce liver changes because of their pharmacokinetic characteristics. Approximately $50 \%$ of the drug is incorporated unchanged in the skeleton, and the other half is non-metabolized and excreted in the urine (Kimmel, 2007; Polyzos et al., 2011).

Bisphosphonates are nephrotoxins and can cause kidney damagez (Weide et al., 2010) . Although the mechanism of injury induction is not completely understood, it is postulated that it occurs through mevalonate pathway inhibition in the tubular cells (Pfister, Atzpodien, Bohrmann, \& Bauss, 2005). The histopathologic changes related bisphosphonates can be represented by focal nephrotoxic necrosis, the presence of apoptosis and the loss of the brush border of the tubular renal cells without causing significant glomerular and interstitial damage (Pfister et al., 2005).

In the present study, the presence of hyaline-cylinders in proximal and distal tubules in all groups that received ZA intravenous administration was observed. These results are corroborated by Pfister et al. (Pfister et al., 2005), who reported the presence of hyaline-cylinders in the kidneys of rats submitted to ibandronate infusions, a second-generation aminobisphosphonate.

This research showed splenic atrophy in animals treated with DEX. It has been demonstrated in the literature that daily injection of $5 \mathrm{mg} /$ $\mathrm{kg}$ (intraperitoneally) dexamethasone for up to 28 days resulted in atrophic white pulp, with no lymphoid follicles, cells undergoing apoptosis and a lower number of lymphocytes (Pereira, Bolzani, Stefani, \& Chalin, 2007). Orzechowski et al. (Orzechowski et al., 2002) showed that mice treated with dexamethasone $(0.5-2 \mathrm{mg} / \mathrm{g}$, by gavage) had a splenic reduction ranging from $45 \%$ in young animals to $52 \%$ in aged rats, which may result from an increased sensitivity of spleen cells to cytotoxic effects from glucocorticoids. These findings confirm the results of current research, where we observed white pulp atrophy and spleen reduction index (data not shown) in control animals treated with dexamethasone and BRONJ 0.4 and $4 \mathrm{mg} / \mathrm{kg}$.

Finally, the anti-inflammatory therapy utilized did not interfere with the development of BRONJ. However, it was observed that chronic treatment with dexamethasone caused dose-dependent leukopenia associated with increases in bacterial colonization and inflammatory infiltrate at tooth extraction sites. Moreover, nimesulide reduced the number of polymorphonuclear neutrophils in the necrotic site without altering other histological features of BRONJ.

\section{Conclusion}

The therapeutic effects of dexamethasone and nimesulide did not prevent the development of BRONJ. Nimesulide was effective in reducing the number of polymorphonuclear neutrophil granulocytes in the alveoli. However, it did not prevent the development of osteonecrosis of the jaw associated with intravenous bisphosphonates. Dexamethasone induced white pulp atrophy of the spleen, and it attenuated leucocytosis post-administration of ZA.

\section{Conflict of interest}

None.

\section{Acknowledgements}

The authors thank the Laboratory of Pharmacology of Inflammation and Cancer, Laboratory of Experimental Oncology, Haematology Laboratory of the Federal University of Ceará, and the Practice of Dental Radiology and Imaging Perboyre Castelo for technical, financial and structural collaboration for the development of this study. The authors also thank the Coordination for the Improvement of Higher Education Personnel (CAPES) for their assistance in this work and for granting scholarships to the graduate student.

\section{References}

Aghaloo, T. L., Kang, B., Sung, E. C., Shoff, M., Ronconi, M., Gotcher, J. E., et al. (2011) Periodontal disease and bisphosphonates induce osteonecrosis of the jaws in the rat. Journal of Bone and Mineral Research, 26, 1871-1882.

Allen, M. R., Reinwald, S., \& Burr, D. (2008). Alendronate reduces bone toughness of ribs without significantly increasing microdamage accumulation in dogs following three years of daily treatment. Calcified Tissue International, 82, 354-360.

Allen, M. R., Chu, T.-M. G., \& Ruggiero, S. L. (2013). Absence of exposed bone following dental extraction in beagle dogs treated with 9 months of high-dose zoledronic acid combined with dexamethasone. Journal of Oral and Maxillofacial Surgery, 71, 1017-1026.

Araújo, F. A. C., Santos, T. S., Morais, H. H. A., Laureano-Filho, J. R., Silva, E. D. O., \& Vasconcellos, R. J. H. (2012). Comparative analysis of preemptive analgesic effect of tramadol chlorhydrate and nimesulide following third molar surgery. Journal of Cranio-Maxillo-Facial Surgery, 40, e346-e349.

Avelar, R. L., Primo, B. T., Vogt, B. F., Silva, E. D. O., Antunes, A. A., Magalhães, M. T. C., et al. (2012). Effect of partially selective cyclooxygenase- 2 inhibitor in the removal of third molars. The Journal of Craniofacial Surgery, 23, e-108-e112.

Baldi, D., Izziotti, A., Bonica, P., Pera, P., \& Pulliero, A. (2009). Degenerative periodontal diseases and oral osteonecrosis: The role of gene-environment interactions. Mutation Research, 667, 118-131.

Barasch, A., Cunha-Cruz, J., Curro, F. A., Hujoel, P., Sung, A. H., Vena, D., et al. (2011). Risk factors for osteonecrosis of the jaws: A case-control study from the CONDOR dental PBRN. Journal of Dental Research, 90, 439-444.

Barros-Silva, P. G., Oliveira, C. C., Brizeno, L. A., Wong, D. V. T., Lima-Júnior, R. C. P., Gonçalves, R. P., et al. (2016). Immune cellular profile of bisphosphonate-related osteonecrosis of the jaw. Oral Diseases, 22, 649-657.

Beato, M. (1989). Gene regulation by steroid hormones. Cell, 56(3), 335-344.

Bi, Y., Gao, Y., Ehirchiou, D., Cao Chunzhang, C., Kikuiri, T., Le, A., et al. (2010) Bisphosphonates cause osteonecrosis of the jaw-like disease in mice. The American Journal of Pathology, 177, 280-290.

Bianchi, M., \& Broggini, M. (2003). A randomized, double blind, clinical trial comparing the efficacy of nimesulide: Celecoxib and rofecoxib in osteoarthritis of the knee. Drugs, 63, 37-46.

Bjarnason, I., \& Thjodleifsson, B. (1999). Gastrointestinal toxicity of non-steroidal antiinflammatory drugs: The effect of nimesulide compared with naproxen on the human gastrointestinal tract. Rheumatology, 38, 24-31.

Bodner, L., Kaffe, I., Littner, M. M., \& Cohen, J. (1993). Extraction site healing in rats. A radiologic densitometric study. Oral Surgery, Oral Medicine, Oral Pathology, 75, 367-372.

Bravo-Cuellar, A., García-Reyes, G., Barba-Barajas, M., Carranco-López, A., \& DominguezRodriguez, J. R. (2003). Modification by nimesulide administration of the phagocytic activity of polymorphonuclears of healthy subjects. Biomedicine \& Pharmacotherapy, $57,434$.

Capsoni, F., Venegoni, E., Minonzio, F., Ongari, A. M., Maresca, V., \& Zanussi, C. (1987). Inhibition of neutrophil oxidative metabolism by nimesulide. Agents and Actions, 21, 121-129.

Chiandussi, S., Biasotto, M., Dore, F., Cavalli, F., Cova, M. A., \& Di Lenarda, R. (2006). Clinical and diagnostic imaging of bisphosphonate-associated osteonecrosis of the jaws. Dentomaxillofacial Radiology, 35, 236-243.

Chiu, C. T., Chiang, W.-F., Chuang, C.-Y., \& Chang, S.-W. (2010). Resolution of oral bisphosphonate and steroid-related osteonecrosis of the jaw-A serial case analysis. Journal of Oral and Maxillofacial Surgery, 68, 1055-1063.

Conte-Neto, N., De Souza Bastos, A., Spolidorio, L. C., Chierici Marcantonio, R. A., \& Marcantonio, E., Jr. (2012). Long-term treatment with alendronate increases the surgical difficulty during simple exodontias - An in vivo observation in Holtzman rats. Head \& Face Medicine, 8, 20-24.

Conte-Neto, N., Spolidorio, L. C., Andrade, C. R., Bastos, A. S., Guimarães, M., \& Marcantonio, E., Jr. (2013). Experimental development of bisphosphonate-related osteonecrosis of the jaws. International Journal of Experimental Pathology, 94, 65-73.

Cullen, L., Kelly, L., Connor, O. S., \& Fitzgerald, D. J. (1998). Selective cyclooxygenase-2 inhibition by nimesulide in man. The Journal of Pharmacology and Experimental Therapeutics, 287, 578-582.

Dapino, P., Ottonello, L., \& Dallegri, F. (1994). The anti-inflammatory drug nimesulide 
inhibits neutrophil adherence to and migration across monolayers of cytokine-activated endothelial cells. Respiration, 61, 336-341.

Del Conte, A., Bernardeschi, P., La Ferla, F., Turrisi, G., D’alessandro, M., Montagnami, F., et al. (2010). Bisphosphonate-induced osteonecrosis of the jaw 32 months after interruption of zoledronate in a patient with multiple myeloma. Journal of Oral and Maxillofacial Surgery, 68, 1179-1182.

Deng, X., Tamai, R., Endo, Y., \& Kiyoura, Y. (2009). Alendronate augments interleukin-1ß release from macrophages infected with periodontal pathogenic bacteria through activation of caspase-1. Toxicology and Applied Pharmacology, 235, 97-104.

Falkenstein, E., Norman, A. W., \& Wehling, M. (2000). Mannheim classification of non genomically initiated (rapid) steroid action(s). Journal of Clinical Endocrinology and Metabolism, 85, 2072-2075.

Favia, G., Pilolli, G. P., \& Maiorano, E. (2009). Histologic and histomorphometric features of bisphosphonate-related osteonecrosis of the jaws: An analysis of 31 cases with confocal laser scanning microscopy. Bone, 45, 406-413.

Halleen, J. M., Räisänen, S., Salo, J. J., Reddy, S. V., Roodman, G. D., Hentunen, T. A., et al. (1999). Intracellular fragmentation of bone resorption products by reactive oxygen species generated by osteoclastic tartrate-resistant acid phosphatase. The Journal of Biological Chemistry, 274, 22907-22910.

Hansen, T., Kirkpatrick, C. J., Walter, C., \& Kunkel, M. (2006). Increased numbers of osteoclasts expressing cysteine proteinase cathepsin $\mathrm{K}$ in patients with infected osteoradionecrosis and bisphosphonate-associated osteonecrosis-A paradoxical observation? Virchows Archiv: An International Journal of Pathology, 449, 448-454.

Hoff, A. O., Toth, B. B., Altundag, K., Johnson, M. M., Warneke, C. L., Hu, M., et al. (2008). Frequency and risk factors associated with osteonecrosis of the jaw in cancer patients treated with intravenous bisphosphonates. Journal of Bone and Mineral Research, 23, 826-836.

Jabbour, Z., Hakim, M. E., Henderson, J. E., \& Albuquerque, R. F. (2014). Bisphosphonates inhibit bone remodeling in the jaw bones of rats and delay healing following tooth extractions. Oral Oncology, 50, 485-490.

Junqueira, L. C. U., \& Junqueira, L. M. M. S. (1983). Técnicas básicas de citologia e histologia (1st ed.). Rio de Janeiro, RJ: Santos Editora.

Kang, B., Cheong, S., Chaichanasakul, T., Bezouglaia, O., Atti, E., Dry, S. M., et al. (2013). Periapical disease and bisphosphonates induce osteonecrosis of the jaws in mice. Journal of Bone and Mineral Research, 28(7), 1631-1640.

Keklikoglu, N. (2004). The localization of Fos B, a member of transcription factor AP-1 family, in rat odontoblasts and pulpal undifferentiated ectomesenchymal cells. Folia Histochemica Et Cytobiologica, 42, 191-193.

Kerachian, M. A., Harvey, E. J., Chow, T. Y., Nahal, A., \& Séguin, C. (2011). A rat model of early stage osteonecrosis induced by glucocorticoids. Journal of Orthopaedic Surgery and Research, 6, 62-68.

Khapra, A. P., \& Rose, S. (2006). Drug injury in the upper gastrointestinal tract: Effects of alendronate. Gastrointestinal Endoscopy Clinics of North America, 16(1), 99-110.

Kimmel, D. B. (2007). Mechanism of action, pharmacokinetic and pharmacodynamic profile, and clinical applications of nitrogen-containing bisphosphonates. Journal of Dental Research, 86, 1022-1033.

Kobayashi, Y., Hiraga, T., Ueda, A., Wang, L., Matsumoto-Nakano, M., Hata, K., et al. (2010). Zoledronic acid delays wound healing of the tooth extraction socket, inhibits oral epithelial cell migration, and promotes proliferation and adhesion to hydroxyapatite of oral bacteria, without causing osteonecrosis of the jaw, in mice. Journal of Bone and Mineral Metabolism, 28, 165-175.

Kopka, A., Janiszewks, S., Szwed, M., Duda, W., \& Bukowska, B. (2011). The effect of alendronate sodium on human erythrocytes. Environmental Toxicology and Pharmacology, 32, 306-314.

Krishnan, A., Arslanoglu, A., Yildirm, N., Silbergleit, R., \& Aygun, N. (2009). Imaging findings of bisphosphonate-related osteonecrosis of the jaw with emphasis on early magnetic resonance imaging findings. Journal of Computer Assisted Tomography, 33, 298-304.

López-Jornet, P., Camacho-Alonso, F., Molina-Miñano, F., Gómez-García, F., \& VicenteOrtega, V. (2010). An experimental study of bisphosphonate-induced jaws osteonecrosis in Sprague-Dawley rats. Journal of Oral Pathology \& Medicine, 39, 697-702.

Licata, A., Calvaruso, V., Cappelo, M., Craxi, A., \& Amasio, P. L. (2010). Clinical course and outcomes of drug-induced liver injury: Nimesulide as the first implicated medication. Digestive and Liver Disease, 42, 143-148.

Ma, Z. Y., \& Wang, Y. X. (2014). Establishment of bisphosphonate-related osteonecrosis of the jaw model in rats and preliminary analysis of its etiological mechanism. Beijing $D a$ Хue Xue Bao, 46, 945-949.

Maahs, M. P., Azambuja, A. A., Campos, M. M., Salum, F. G., \& Cherubini, K. (2011). Association between bisphosphonates and jaw osteonecrosis: A study in Wistar rats. Head \& Neck, 33, 199-207.

Marino, K. L., Zakhary, I., Abdelsayed, R. A., Carter, J. A., O'neill, J. O., Khashaba, R. M., et al. (2012). Development of a rat model of bisphosphonate-related osteonecrosis of the jaw (BRONJ). The Journal of Oral Implantology, 38, 511-528.

Marx, R. E., Sawatari, Y., Fortin, M., \& Broumand, V. (2005). Bisphosphonate-induced exposed bone (osteonecrosis/osteopetrosis) of the jaws: Risk factors, recognition, prevention, and treatment. Journal of Oral and Maxillofacial Surgery, 63, 1567-1575.

Mello, S. B. V., Laurindo, I. M. M., \& Cossermelli, W. (1994). Action of the 4-nitro-2 phenoximethanesulphonanilide (Nimesulide) on neutrophil chemotaxis and superoxid production. São Paulo Medical Journal, 112, 489-494.

Menezes, S. A. F., \& Cury, P. R. (2010). Efficacy of nimesulide versus meloxicam in the control of pain: Swelling and trismus following extraction of impacted lower third molar. International Journal of Oral and Maxillofacial Surgery, 39, 580-584.

Mingatto, F. E., Rodrigues, T., Pigoso, A. A., Uyemura, S. A., Curtis, C., \& Santos, A. C. (2002). The critical role of mitochondrial energetic impairment in the toxicity of nimesulide to hepatocytes. Journal of Pharmacology and Experimental Therapeutics, 303, 601-607.

Mizuno, H., Uemura, K., Moriyama, A., Wada, Y., Asai, K., Kimura, S., et al. (1997). Glucocorticoid induced the expression of mRNA and the secretion of lipocortin 1 in rat astrocytoma cells. Brain Research, 746, 256-264.

Mozzati, M., Martinasso, G., Maggiora, M., Scoletta, M., Zambelli, M., Carossa, S., et al. (2013). Oral mucosa produces cytokines and factors influencing osteoclast activity and endothelial cell proliferation, in patients with osteonecrosis of jaw after treatment with zoledronic acid. Clinical Oral Investigation, 17, 1259-1266.

Orzechowski, A., Ostaszewski, P., Wilczak, J., Jank, M., Bałasinska, B., Wareski, P., et al. (2002). Rats with a glucocorticoid-induced catabolic state show symptoms of oxidative stress and spleen atrophy: The effects of age and recovery. Journal of Veterinary Medicine, 49, 256-263.

Ottonello, L., Dapino, P., Pastorino, G., \& Dallegri, F. (1992). Inhibition of the neutrophil oxidative response induced by the oral administration of nimesulide in normal volunteers. Journal of Clinical Laboratory Immunology, 37, 91-96.

Pazianas, M. (2011). Osteonecrosis of the jaw and the role of macrophages. Journal of the National Cancer Institute, 103, 232-240.

Pereira, A. L. C., Bolzani, F. C. B., Stefani, M., \& Chalin, R. (2007). Uso sistêmico de corticosteróides: Revisão da literatura. Medicina Cutanea Ibero-Latino-Americana, 35, 35-50.

Pfister, T., Atzpodien, E., Bohrmann, B., \& Bauss, F. (2005). Acute renal effects of intravenous bisphosphonates in the rat. Basic \& Clinical Pharmacology \& Toxicology, 97, 374-381.

Polyzos, S. A., Kountouras, J., Anastasilakis, A. D., Litsas, I., Kita, M., Arsos, G., et al. (2011). Zoledronic acid-induced transient hepatotoxicity in a patient effectively treated for Paget's disease of bone. Osteoporosis International, 22, 363-367.

Pozzi, S., Marcheselli, R., Sacchi, S., Baldini, L., Angrilli, F., Pennese, E., et al. (2007) Bisphosphonate-associated osteonecrosis of the jaw: A review of 35 cases and an evaluation of its frequency in multiple myeloma patients. Leukemia \& Lymphoma, 48, 56-64.

Ragot, J. P., Giorgi, M., Marinoni, M., Macchi, M., Mazza, P., Rizzo, S., et al. (1994). Acute activity of nimesulide in the treatment of pain after oral surgery-double-blind: Placebo and mefenamic acid controlled study. European Journal Clinical Research, 5, 39-50.

Reigner, B. G., \& Blesch, K. S. (2002). Estimating the starting dose for entry into humans: Principles and practice. European Journal of Clinical Pharmacology, 57, 835-845.

Roelofs, A. J., Thompson, K., Ebetino, F. H., Rogers, M. J., \& Coxon, F. P. (2010). Bisphosphonates: Molecular mechanism of action and effects on bone cells: Monocytes and macrophages. Current Pharmaceutical Design, 16, 2950-2960.

Ruggiero, S., Dodson, T. B., Assael, L. A., Landesberg, R., Marx, R. E., \& Mehrotra, B. (2009). American Association of Oral and Maxillofacial Surgeons position paper on bisphosphonate-related osteonecrosis of the jaw-2009 update. Australian Endodontic Journal, 35, 119-130.

Ruggiero, S. L., Dodson, T. B., Fantasia, J., Goodday, R., Aghaloo, T., Mehrotra, B., et al. (2014). American association of oral and maxillofacial surgeons position paper on medication-related osteonecrosis of the jaw-2014 update. Journal of Oral and Maxillofacial Surgery, 72, 1938-1956.

Shah, A. A., Thjodleifsson, B., Murray, F. E., Sightorsson, G., Oddson, E., Gudjonsson, H., et al. (2001). A randomised, double blind, double dummy: Crossover study of the effects of nimesulide and naproxen on the gastrointestinal tract and na in vivo assessment of their selectivity for cyclooxygenase 1 and 2. Gut, 48, 339-348.

Silva, P. G. B., Ferreira-Junior, A. E. C., Teofilo, C. R., Barbosa, M. C., Lima-Júnior, R. C. P., Sousa, F. B., et al. (2015). Effect of different doses of zoledronic acid in establishing of bisphosphonate-related osteonecrosis. Archives of Oral Biology, 60, 1237-1245.

Sonis, S. T., Watkins, B. A., Lyng, G. D., Lerman, M. A., \& Anderson, K. C. (2009). Bony changes in the jaws of rats treated with zoledronic acid and dexamethasone before dental extractions mimic bisphosphonate-related osteonecrosis in cancer patients. Oral Oncology, 45, 164-172.

Tian, L., Wen, Q., Dang, X., Fan, L., \& Wang, K. (2014). Immune response associated with Toll-like receptor 4 signaling pathway leads to steroid-induced femoral head osteonecrosis. BME Musculoskeletal Disorders, 15, 18-28.

Tompkins, K. A. (2016). The osteoimmunology of alveolar bone loss. Connective Tissue Research, 57, 69-90.

Tsao, C., Darby, I., Ebeling, P. R., Walsh, K., O'brien-Simpson, N., Reynolds, E., et al. (2013). Oral health risk factors for bisphosphonate-associated jaw osteonecrosis. Journal of Oral and Maxillofacial Surgery, 71, 1360-1366.

Walter, C., Klein, M. O., Pabst, A., Nawas, A., Duschner, H., \& Ziebart, T. (2010). Influence of bisphosphonates on endothelial cells, fibroblasts, and osteogenic cells. Clinical Oral Investigation, 14, 35-41.

Weide, R., Köppler, H., Antràsl, L., Smith, M., Chang, E. Mph, Green, J., et al. (2010). Renal toxicity in patients with multiple myeloma receiving zoledronic acid vs. ibandronate: A retrospective medical records review. Journal of Cancer Research and Therapeutics, 6, 31-35.

Zhu, M., Liang, R., Pan, L-H., Huang, B., Quian, W., Zhong, J-H., Zheng, W-W. \& Li, C-L. Zoledronate for metastatic bone disease and pain: a meta-analysis of randomized clinical trials. Pain Medicine, 14, 257-264. 